\title{
MicroRNA-145 regulates the proliferation, migration and invasion of human primary colon adenocarcinoma cells by targeting MAPK1
}

\author{
YONG YANG ${ }^{1}$, XIAO-JIA LI ${ }^{1}$, PENG LI $^{2}$ and XIU-TIAN GUO ${ }^{1}$ \\ ${ }^{1}$ Department of Anus and Intestine; ${ }^{2}$ College of Clinical Medicine, \\ Shanghai Hospital of Traditional Chinese Medicine, Shanghai 200071, P.R. China
}

Received June 8, 2018; Accepted September 27, 2018

DOI: $10.3892 / \mathrm{ijmm} .2018 .3904$

\begin{abstract}
Primary colon adenocarcinoma is responsible for high rates of mortality worldwide. The late diagnosis and lack of reliable biomarkers and therapeutic targets forms a bottleneck in the treatment of colon cancer. In the present study, the therapeutic potential of an important microRNA (miR), namely miR-145, was investigated in primary colon adenocarcinoma cells. The results revealed that the expression of miR-145 was significantly $(\mathrm{P}<0.05)$ downregulated in colon adenocarcinoma cells and the ectopic expression of miR-145 in colon cancer inhibited proliferation by promoting the apoptosis of SW480 primary colon adenocarcinoma cells. Furthermore, bioinformatics analysis revealed that miR-145 exerts its effected by targeting mitogen-activated protein kinase (MAPK) in SW480 cells. This was confirmed by expression analysis wherein the expression of MAPK1 was significantly $(\mathrm{P}<0.05)$ upregulated in the primary colon adenocarcinoma cells and the ectopic expression of miR-145 inhibited the expression of MAPK1. By contrast, the silencing of MAPK1 had similar effects on the proliferation, migration and invasion of SW480 cells as that of the overexpression of miR-145. Furthermore, it was observed that the inhibition of miR-145 did not reverse the effects of MAPK1 silencing on SW480 cells. However, the overexpression of MAPK1 led to considerable reversal of the effects of the overexpression of $\mathrm{miR}-145$ on the proliferation, migration and invasion of SW480 cells. The effects of the overexpression of miR-145 were also evaluated in vivo in xenografted mice and it was observed that the overexpression of miR-145 also inhibited tumor growth and volume in vivo. Taken together, it was concluded that miR-145 may prove to be an important therapeutic target for colon cancer.
\end{abstract}

Correspondence to: Dr Xiu-Tian Guo, Department of Anus and Intestine, Shanghai Hospital of Traditional Chinese Medicine, 274 Zhijiang Middle Road, Shanghai 200071, P.R. China

E-mail: toddjparkerpz@yahoo.com

Key words: microRNA, colon adenocarcinoma, mitogen-activated protein kinase 1 , apoptosis, metastasis

\section{Introduction}

Primary colon adenocarcinoma is one of the leading causes of cancer-associated mortality worldwide (1). Age, sex, diet and genetic factors have implications in the development of colon adenocarcinoma (2). Although, a number of treatment approaches, including surgery and chemotherapy, are used for the management of this type of cancer, the five-year survival rates remain poor (3). The late diagnosis and unavailability of reliable biomarkers and therapeutic targets form a major obstacle in the treatment of colon adenocarcinoma (4). MicroRNAs are small RNA molecules, which do not code for any protein and have been found to be involved in a number of cellular processes (5). MicroRNAs have also been implicated in the development of a number of diseases, which include, but are not limited to, cancer (6). The expression of several microRNAs has been reported to be dysregulated in cancer cells and microRNAs are being considered as important therapeutic targets/agents (7). MicroRNA (miR)-145 is one such microRNA, which has been found to be dysregulated in a number of cancer types. For example, the expression of miR-145 has been reported to be significantly downregulated in gastric and prostate cancer $(1,8)$. Furthermore, miR-145 has been shown to be involved in the regulation of proliferation, migration and invasion of cancer cells; for example, Sachdeva and Mo reported that miR-145 inhibits the proliferation and metastasis of breast cancer cells (9). However, the role and therapeutic potential of miR-145 have not been investigated in primary colon adenocarcinoma cells. In the present study, the expression of miR-145 in normal and primary colon adenocarcinoma cells was examined. It was found that the expression of miR-145 was significantly downregulated in all colon adenocarcinoma cell lines. Furthermore, the ectopic expression of miR-145 in colon adenocarcinoma cells inhibited their proliferation via the induction of apoptosis and suppression of migration and invasion by targeting mitogen-activated protein kinase 1 (MAPK1). The expression of MAPK1 has been reported to be upregulated in a number of types of cancer and several anticancer agents have been shown to target MAPK1 (10). In the present study, the effects of the overexpression of miR-145 were also evaluated in xenografted mice and it was observed that miR-145 inhibited tumor growth 
in vivo. Taken together, the results suggested that miR-145 may prove to be an important therapeutic target/agent for the management of colon adenocarcinoma.

\section{Materials and methods}

Cell lines and culture conditions. The normal colon cell line (CCD-18Co) and primary colon adenocarcinoma cell lines (Hs255.T, SW480, Hs257.T, HT29, LS174T, SNU-C1, and DLD-1) were procured from American Type Culture Collection (Manassas, VA, USA). All cell lines were maintained in Dulbecco's modified Eagle's medium supplemented with $10 \%$ fetal bovine serum (Thermo Fisher Scientific, Inc., Waltham, MA, USA), antibiotics (100 U/ml penicillin and $100 \mu \mathrm{g} / \mathrm{ml}$ streptomycin), and $2 \mathrm{mM}$ glutamine. The cells were cultured in a $\mathrm{CO}_{2}$ incubator (Thermo Fisher Scientific, Inc.) at $37^{\circ} \mathrm{C}$ with $98 \%$ humidity and $5 \% \mathrm{CO}_{2}$.

Reverse transcription-quantitative polymerase chain reaction (RT-qPCR) analysis. The total RNA was extracted from the primary colon adenocarcinoma cell lines (Hs255.T, SW480, Hs257.T, HT29, LS174T, SNU-C1, DLD-1) and normal cell line (CCD-18Co) with the assistance of RNeasy kits (Qiagen, Inc., Valencia, CA, USA). To reverse transcribe the cDNA, the Omniscript RT (Qiagen, Inc.) was employed using $1 \mu \mathrm{g}$ of the extracted RNA. The cDNA was then used as a template for RT-qPCR analysis with the assistance of the Taq PCR Master Mix kit (Qiagen, Inc.) according to the manufacturer's protocol. The reaction mixture consisted of $20 \mu \mathrm{l}$ containing $1.5 \mathrm{mM} \mathrm{MgCl} 2,2.5$ units Taq DNA Polymerase, $200 \mu \mathrm{M}$ dNTP, $0.2 \mu \mathrm{M}$ of each primer and $0.5 \mu \mathrm{g}$ DNA. The cycling conditions were as follows: $95^{\circ} \mathrm{C}$ for $20 \mathrm{sec}$, followed by 40 cycles of $95^{\circ} \mathrm{C}$ for $15 \mathrm{sec}$, and $58^{\circ} \mathrm{C}$ for $1 \mathrm{~min}$. GAPDH was used as an internal control and the relative quantification $\left(2^{-\Delta \Delta \mathrm{Cq}}\right)$ method was used to evaluate the quantitative variation between the samples as described previously (11). The primers used in the present study are presented in Table I.

Cell transfection. When the primary colon adenocarcinoma SW480 cells reached $80 \%$ confluence, they were transfected with mimics-negative control (NC; 5'-GUAGGAGUAGUG AAAGGCC-3'), miR-145 mimics (5'-GUCCAGUUUUCC CAGGAAUCCCU-3'), or miR-145 inhibitor (5'-AAGGGA UUCCUGGGAAACUGGAC-3'), from Shanghai GenePharma (Shanghai, China; 10 pmol), small interfering (si)-negative control (si-NC) (5'-CGA ACUCACUGGUCUGACC-3'), siRNA-MAPK1 (5'-AGUUCGAGUAUACUUCAAGUU-3') and pcDNA-MAPK1 ( $2 \mu \mathrm{g}$, Taijin Saier Biotechnology, China) with Lipofectamine 2000 (Invitrogen; Thermo Fisher Scientific, Inc.) as per the manufacturer's protocol.

Cell viability and colony formation assays. The cell viability of the primary SW480 adenocarcinoma cells was assessed using theWST-1 colorimetric assay. Briefly, the SW480 primary adenocarcinoma cells were seeded in 96-well plates at the density of $2 \times 10^{5}$ cells/well. The cells were then incubated with WST- 1 at $37^{\circ} \mathrm{C}$ for $4 \mathrm{~h}$. The absorbance at $450 \mathrm{~nm}$ was then measured using a microplate reader to determine the viability of primary adenocarcinoma cells. For assessment of the colony formation potential of the SW480 cells, the cells were collected at the exponential phase of growth and were then counted using a hemocytometer. The platting of the transfected cells was performed at 200 cells/well. The plates were then maintained at $37^{\circ} \mathrm{C}$ for 6 days. Following incubation of the cells for 6 days, they were subjected to washing with PBS and fixation with methanol. The SW480 cells were then stained with crystal violet, and analyzed by light microscopy (Olympus Corporation, Tokyo, Japan).

Detection of apoptosis. The nuclear morphology of the SW480 primary adenocarcinoma cells was assessed by fluorescence microscopy following subjecting the cells to DAPI, AO/EB, Annexin V/PI staining for determination of the apoptotic cell populations, as described previously $(12,13)$.

Target identification and dual luciferase assay. To identify the target, miR-145 was subjected to the online software TargetScan version 7.2 (http://www.targetscan.org). For the luciferase reporter assay, the binding sites of the wild-type (WT) and the mutated (MUT) MAPK1 3'-UTR were cloned into the downstream region of the luciferase gene in the PGL3-REPORT luciferase vector (Invitrogen; Thermo Fisher Scientific, Inc.). The cells were then subjected to co-transfection with the WT and MUT PGL3-MAPK1-3' UTR vectors and the miR-145 mimics. Finally, the luciferase activity was determined using a Luciferase Reporter Assay kit (Promega Corporation, Madison, WI, USA) as per the manufacturer's protocol, and Renilla luciferase activity was used to normalize the data.

Cell migration and invasion assay. The cell migration and invasion of the SW480 cells were investigated using the method of Wang et al, as described previously (14).

Western blot analysis. The primary colon adenocarcinoma cells were transfected with different constructs and then centrifuged at $400 \mathrm{~g}$ for $5 \mathrm{~min}$ at $4^{\circ} \mathrm{C}$, subjected to PBS washing and then subsequently lysed in RIPA buffer containing $50 \mathrm{mM}$ Tris-HCl (pH 7.4), $150 \mathrm{mM} \mathrm{NaCl}, 1 \%$ Triton X-100, 0.1\% SDS, $5 \mathrm{mM}$ EDTA, $30 \mathrm{mM} \mathrm{Na}_{2} \mathrm{HPO}_{4}, 50 \mathrm{mM} \mathrm{NaF}, 0.5 \mathrm{mM} \mathrm{NaVO}_{4}$, $2 \mathrm{mM}$ phenyl methylsulfonyl fluoride, and $10 \%$ protease cocktail inhibitor. This was followed by incubation for $30 \mathrm{~min}$ and the supernatant containing proteins were harvested by centrifugation at $13,000 \mathrm{~g}$ for $15 \mathrm{~min}$ at $4^{\circ} \mathrm{C}$. Protein concentration was determined by the Bradford method (15) and stored at $-80^{\circ} \mathrm{C}$. For western blot analysis, equal quantities of proteins $(50 \mu \mathrm{g})$ were loaded and resolved on an SDS-polyacrylamide gel $(10 \%)$. Following gel electrophoresis, the proteins were transferred onto polyvinylidene difluoride membranes. The membranes were blocked in blocking buffer $(10 \mathrm{mM}$ Tris- $\mathrm{HCl}$, $150 \mathrm{mM} \mathrm{NaCl}, 0.1 \%$ Tween-20) containing 5\% non-fat milk for $1 \mathrm{~h}$ at room temperature and blotted with MAPK1 primary antibodies (1:1,000; anti-MAPK1, cat. no. sc-136288, Santa Cruz Biotechnology, Inc., Dallas, TX, USA) overnight at $4^{\circ} \mathrm{C}$. The blots were washed in TBS, incubated with horseradish peroxidase-conjugated secondary antibody $(1: 1,000$; cat. no. sc-516087, Santa Cruz Biotechnology, Inc.) for $1 \mathrm{~h}$ at $4^{\circ} \mathrm{C}$, washed again three times with TBS and chemiluminescence was captured on hyperfilm following incubating the blots in ECL plus solution. 
Table I. List of primers used for reverse transcriptionquantitative polymerase chain reaction analysis.

\begin{tabular}{ll}
\hline Gene & \multicolumn{1}{c}{ Primer sequence } \\
\hline MAPK1 & F: 5'-TGGATTCCCTGGTTCTCTCTAAAG-3' \\
& R: 5'-GGGTCTGTTTTCCGAGGATGA-3' \\
miR-145 & F:5'-ACACTCCAGCTGGGCAGGTCAAAAGGGTCC-3' \\
& R: 5'-TGCGGGTGCTCGCTTCGGCAGC-3' \\
GAPDH & F: 5'-GAAGGTGAAGGTCGGAGTC-3' \\
& R: 5'-GAAGATGGTGATGGGATTTC-3'
\end{tabular}

MAPK1, mitogen-activated protein kinase 1; miR, microRNA; F, forward; $\mathrm{R}$, reverse.

Mice xenografts. The 4-week-old male BALB/c nude mice weighing $18 \pm 2 \mathrm{~g}$ were obtained from the animal holding capacity of the Shanghai Hospital of Traditional Chinese Medicine and maintained per the National Institutes of Health standards for the care and use of laboratory animals. The study was approved and supervised by the Ethics Committee of Shanghai Hospital of Traditional Chinese Medicine (Shanghai, China) under approval number ECSHT-AS345/2017. The animals were provided free access to a pellet diet, and water was freely available. The animals were maintained in well-ventilated rooms with a controlled environment of a day: night light/dark cycle and temperature of $28 \pm 2^{\circ} \mathrm{C}$. The mice were randomly divided into two groups ( $\mathrm{n}=15$ per group). SW480 cell clones $\left(\sim 1.0 \times 10^{7}\right.$ cells/mouse $)$, were subcutaneously injected with miR-145 mimics or miR-NC into the back of the mice. The mice were sacrificed by deep anesthesia with isoflurane at different time points and sacrifice was verified by monitoring breathing and heartbeat. The tumors were extracted and tumor weight and volume were measured. The tumor volume was determined using the following formula: $\mathrm{V}=(\mathrm{W} \times \mathrm{W} \times \mathrm{L}) / 2$; where ' $\mathrm{V}$ ' is the volume, ' $\mathrm{W}$ ' is the width and ' $\mathrm{L}$ ' is the length of the tumor. RNA and proteins were extracted from the tumor tissues for further experimentation. Each group included five mice and experiments were repeated three times.

Statistical analysis. The experiments were performed three times and the values presented as the mean \pm standard deviation of the three replicates. Student's t-test (for comparison between two samples) and one-way analysis of variance followed by Tukey's test (for comparison between more than two samples) were used for statistical analysis using GraphPad Prism software (version 7; GraphPad Software, Inc., La Jolla, CA, USA). $\mathrm{P}<0.05$ was considered to indicate a statistically significant difference.

\section{Results}

Expression analysis of miR-145 in primary colon adenocarcinoma cells. The expression of miR-145 was examined in seven primary colon adenocarcinoma cell lines (Hs255.T, SW480, Hs257.T, HT29, LS174T, SNU-C1, DLD-1) and one normal cell line (CCD-18Co) by RT-qPCR analysis.
The results revealed that the expression of miR-145 was significantly downregulated in all colon adenocarcinoma cell lines (Hs255.T, SW480, Hs257.T, HT29, LS174T, SNU-C1, and DLD-1). The expression of miR-145 was up to 10 -fold lower in the Hs255.T, SW480, Hs257.T, HT29, LS174T, SNU-C1 and DLD-1 adenocarcinoma cell lines, in comparison to the normal CCD-18Co cell line. The most marked downregulation was found in the Hs257.T cell line, whereas the least downregulated expression of miR-145 was found in the SNU-C1 cell line (Fig. 1A).

Ectopic expression of miR-145 inhibits the proliferation of SW480 cells. Due to the low expression of miR-145 in all the colon adenocarcinoma cell lines, the SW480 cell line was selected for the overexpression experiments to examine the role of miR-145 on cell proliferation. The overexpression of miR-145 was induced by transfection of the SW480 cells with miR-145 mimics, which resulted in a 5.6-fold increase in the expression of miR-145 as revealed by the RT-qPCR analysis (Fig. 1B). Furthermore, it was found that the overexpression of miR145 caused significant $(\mathrm{P}<0.05)$ inhibition of the proliferation of the SW480 cells (Fig. 1C). These results were validated by the colony formation assay, which showed that the overexpression of miR-145 suppressed the colony formation potential of the SW840 cells (Fig. 1D). To investigate the underlying mechanism, the cells were subjected to DAPI and $\mathrm{AO} / \mathrm{EB}$ staining. It was found that the ectopic expression of miR-145 in cells triggered apoptotic death of the SW480 primary colon adenocarcinoma cells (Fig. 1E and F). The percentage of the apoptotic cell populations was estimated by Annexin V/PI staining and it was found that, compared with $2.61 \%$ apoptotic cells in the miR-NC-transfected SW480 cells, the apoptotic cell percentage was $39.6 \%$ in the miR-145 mimics-transfected cells. Taken together these results indicated that the ectopic expression of miR-145 inhibited the proliferation of SW480 colon adenocarcinoma cells by triggering apoptotic cell death.

Ectopic expression of miR-145 suppresses the migration and invasion of SW480 cells. The Transwell cell assays were used to assess the effect of the overexpression of miR-145 on the migration and invasion of the SW480 colon adenocarcinoma cells. The results revealed that the ectopic expression of miR-145 significantly $(\mathrm{P}<0.05)$ suppressed the migration and invasion potential of the SW480 colon adenocarcinoma cells (up to 60\%; Fig. 2A). To determine whether the effects of the overexpression of miR-145 on the migration and invasion were specific to the cancer cells, miR-145 was also overexpressed in the normal CCD-18Co cells. The results indicated that the overexpression of miR-145 had no significant effect on the migration and invasion of the normal CCD-18Co cells (Fig. 2B). Taken together, these results suggested that miR-145 specifically inhibited the migration and invasion of the colon adenocarcinoma cells.

miR-145 exerts its effects on SW480 cells by targeting MAPK1. The bioinformatics analysis with TargetScan revealed several targets for miR-145, which included MAPK1, signal transducer and activator of transcription, mucin 1 , mucin 13 , and insulin receptor substrate 1 (data not shown). However, no 
A
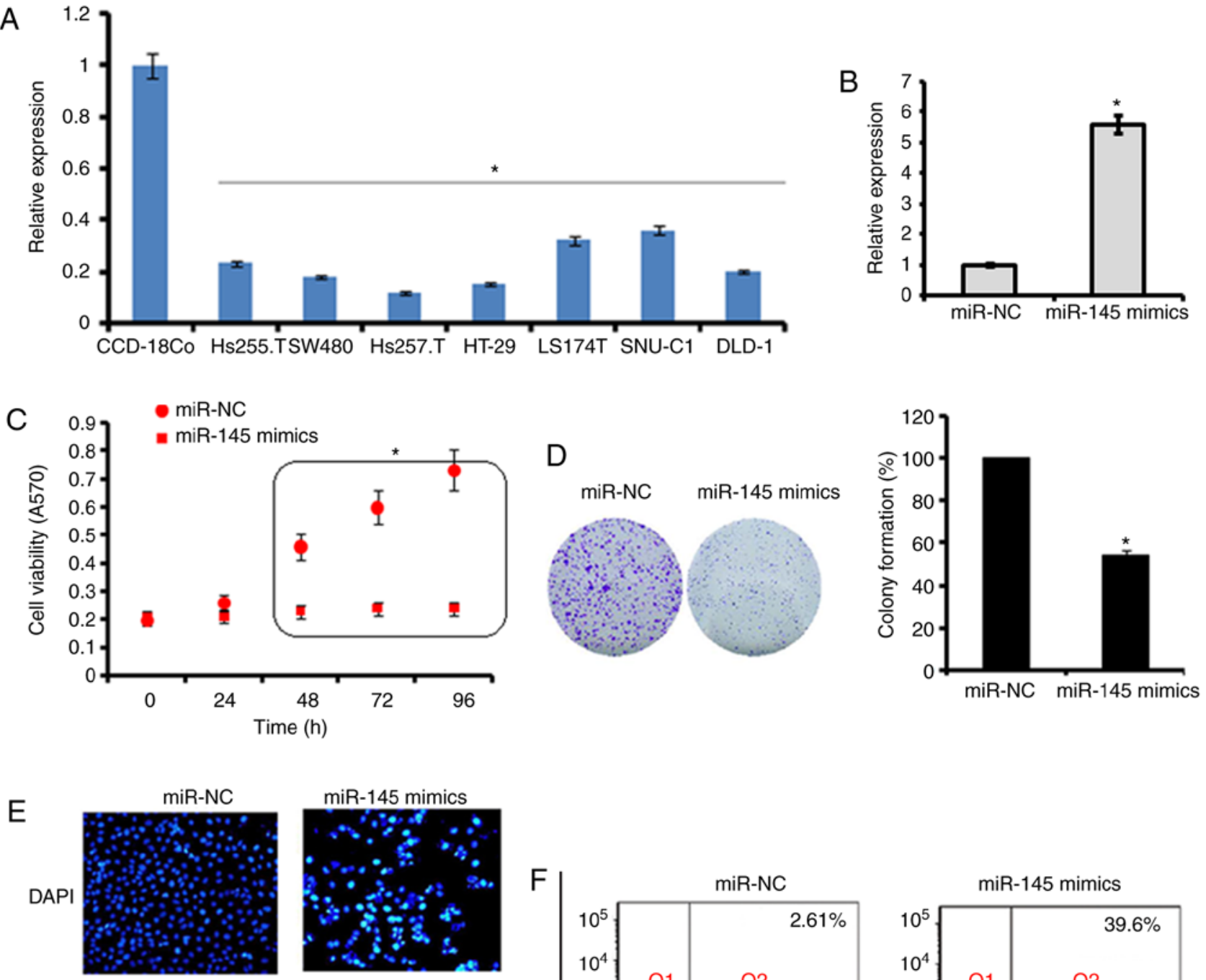

miR-145 mimics
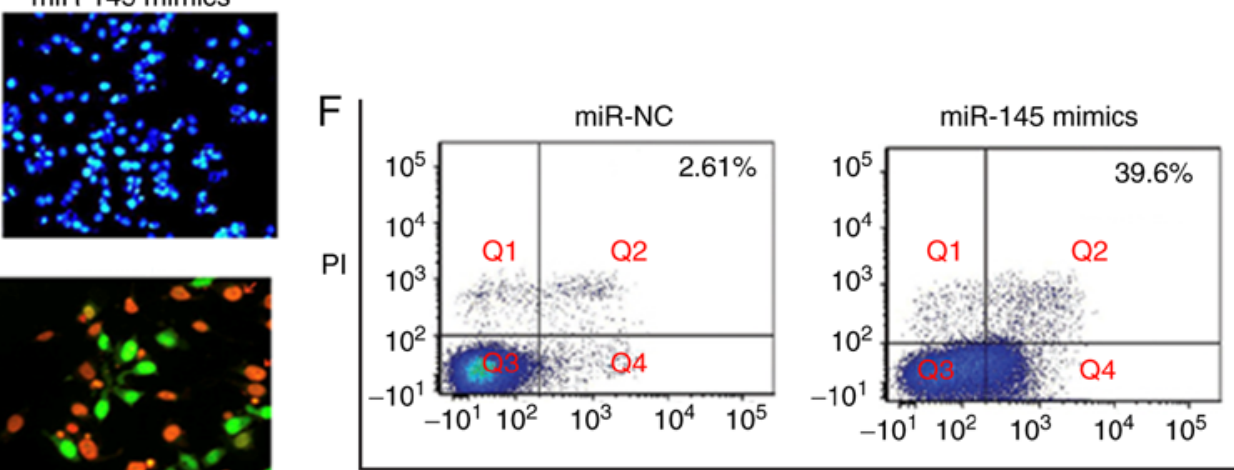

Annexin V-FITC

Figure 1. Analysis of the expression of miR-145. (A) Expression of miR-145 in primary colon adenocarcinoma cell lines and normal CCD-18Co cells (B) Expression of miR-145 in miR-145 mimics- or miR-NC-transfected SW480 cells was determined by reverse transcription-quantitative polymerase chain reaction analysis. (C) Proliferation of SW480 cells and CCD-18Co cells transfected with miR-NC and miR-145 mimics. (D) Colony formation of miR-NC- and miR-145 mimics-transfected SW480 cells. Apoptosis was detected in miR-NC- and miR-145 mimics-transfected SW480 cells by (E) DAPI and (F) Annexin V/PI staining. The values are expressed as the mean \pm standard deviation of three biological replicates ( $\mathrm{P}<0.05)$. miR, microRNA; NC, negative control (magnification, x200).

studies have investigated MAPK1 as the target of miR-145, therefore, MAPK1 was selected for further investigation in the present study (Fig. 3A). Firstly, the expression of MAPK1 was investigated in the Hs255.T, SW480, Hs257.T, HT29, LS174T, SNU-C1, and DLD-1colon adenocarcinoma cell lines and the CCD-18Co normal cell line. It was found that the expression of MAPK1 was significantly upregulated in the colon adenocarcinoma cell lines (Fig. 3B). However, the ectopic expression of miR-145 in SW480 colon adenocarcinoma cells, caused marked downregulation of the expression of MAPK1 (Fig. 3C). The dual luciferase reporter assay further confirmed MAPK1 to be the target of miR-145 (Fig. 3D). To further investigate the effect of MAPK1 on the proliferation, migration and invasion of the
SW480 cells, its expression was silenced by transfection of the SW480 cells with si-MAPK1. The reduction in the expression of the si-MAPK1-transfected SW480 cells was confirmed by RT-qPCR analysis, which showed a 7.2-fold decrease in the expression of MAPK1 in the SW480 cells (Fig. 3E). The effect of MAPK1 suppression on the proliferation of SW480 cells was also investigated. It was found that the silencing of MAPK1 also inhibited the proliferation of SW480 cells by triggering apoptosis (Fig. 3F-H). Similar to the overexpression of miR-145, MAPK1 silencing suppressed the migration and invasion of the SW480 colon adenocarcinoma cells, further confirming MAPK1 as the target of miR-145 (Fig. 4). Subsequently, to overrule the possibility of the involvement of 
A
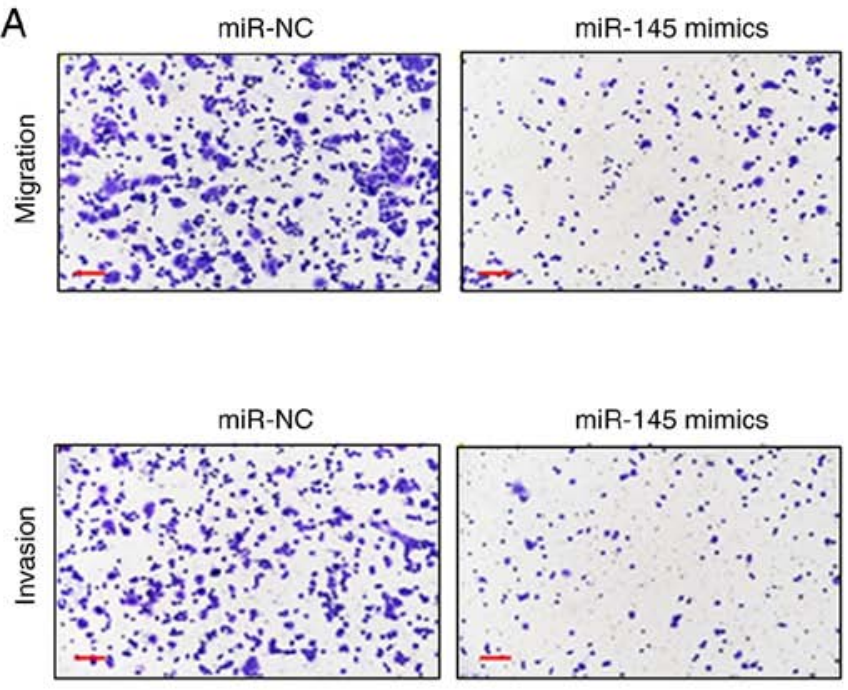

B
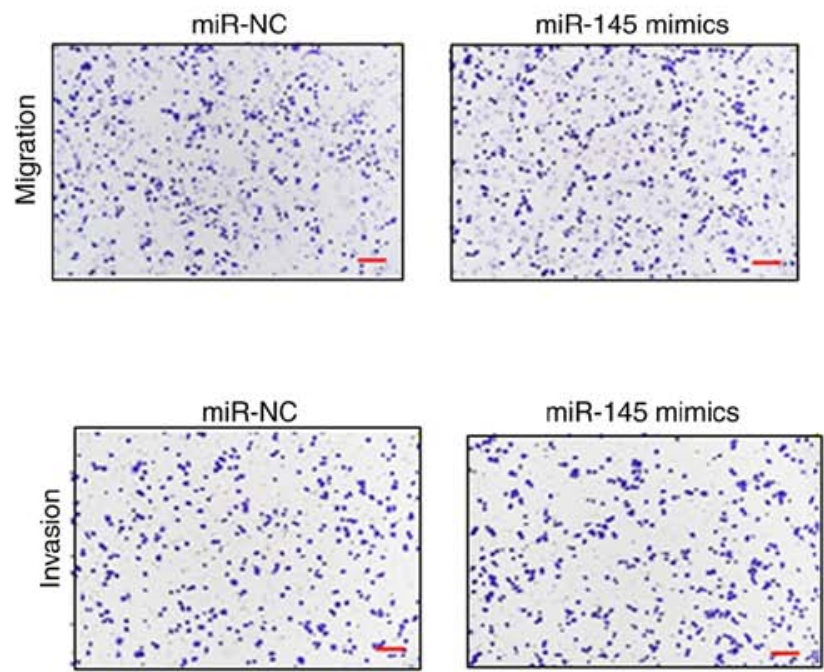
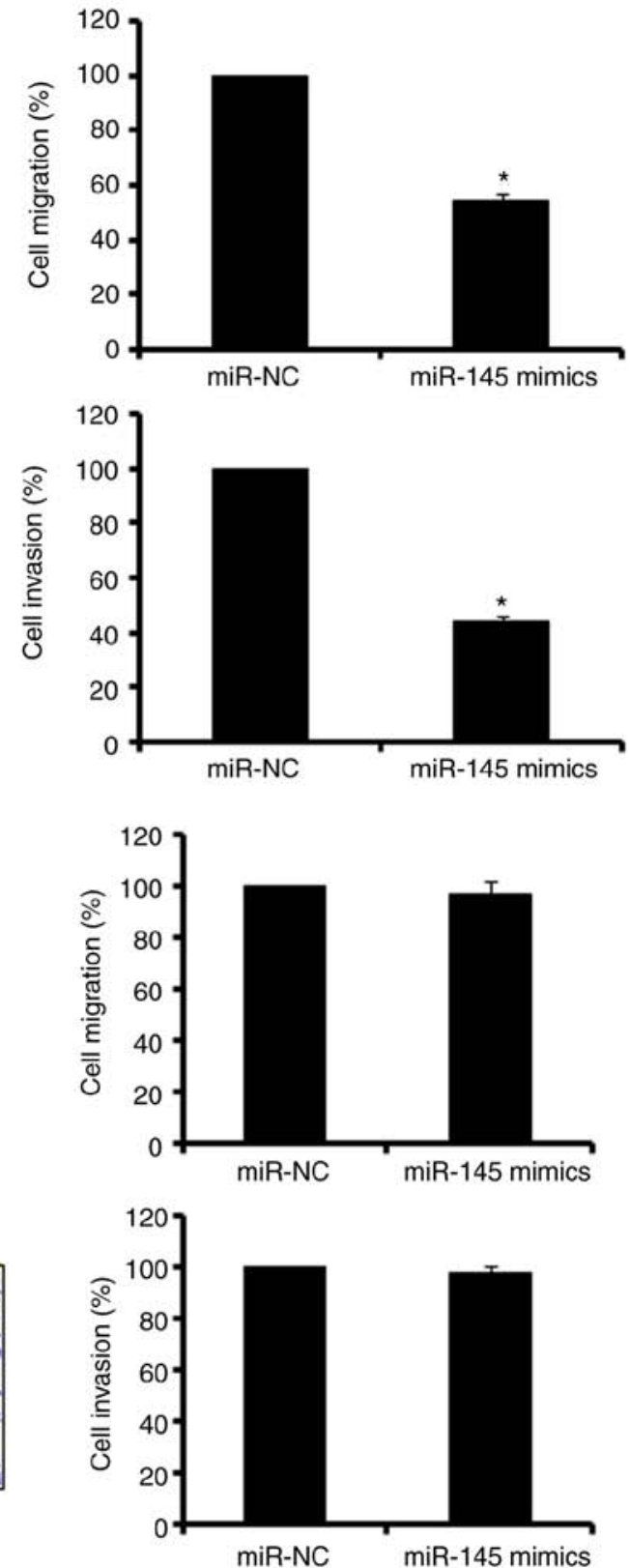

Figure 2. Effect of the overexpression of miR-145 on cell migration and invasion. (A) SW480 primary colon adenocarcinoma cell migration and invasion. (B) CCD-180 normal cell migration and invasion. Scale bars, $100 \mu \mathrm{m}$. The values are expressed as the mean \pm standard deviation of three biological replicates $\left({ }^{*} \mathrm{P}<0.05\right)$. miR, microRNA; NC, negative control.

any other target in the miR-145-induced effects, the present study investigated whether co-transfection of the SW480 cells with si-MAPK1 and miR-145 inhibitor, was able to rescue the effects of MAPK1 silencing on SW480 cell proliferation and migration. It was observed that the inhibition of miR-145 did not reverse the effects of MAPK1 silencing on the proliferation, migration and invasion of SW480 cells (Fig. 5A-C). However, the overexpression of MAPK1 reversed the effects of the miR-145 mimics on the proliferation and migration and invasion of the SW480 cells (Fig. 6A-C).

miR-145 inhibits tumor growth in vivo. To examine the effects of miR-145 effects in vivo, a colon adenocarcinoma xenograft model using BALB/c nude mice was developed. The mice were sacrificed and the tumors were harvested (Fig. 7A). The
RT-PCR assays showed that xenografts of the miR-145 mimic group exhibited higher expression of miR-145 compared with the miR-NC group, indicating the successful overexpression of miR-145 in the colon adenocarcinoma xenograft (Fig. 7B). In addition, the monitoring of tumor volume and the measurement of tumor weights revealed that the overexpression of miR-145 significantly inhibited the growth of tumor xenografts, leading to decreased tumor volumes and weights (Fig. 7C and D).

\section{Discussion}

Colon adenocarcinoma has a high rate of mortality owing to it late diagnosis and lack of reliable biomarkers and therapeutic targets (16). miRNAs are 20 nucleotide long non-coding RNA molecules, which have been found to have vital functions 


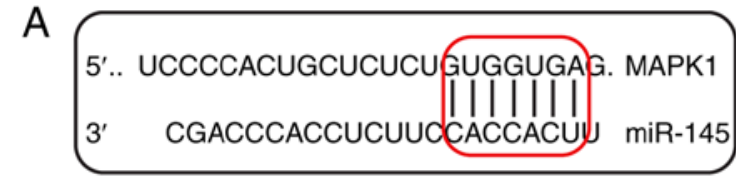

C

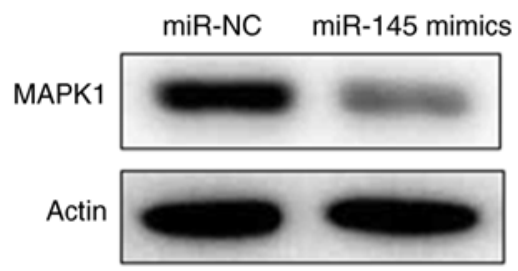

E

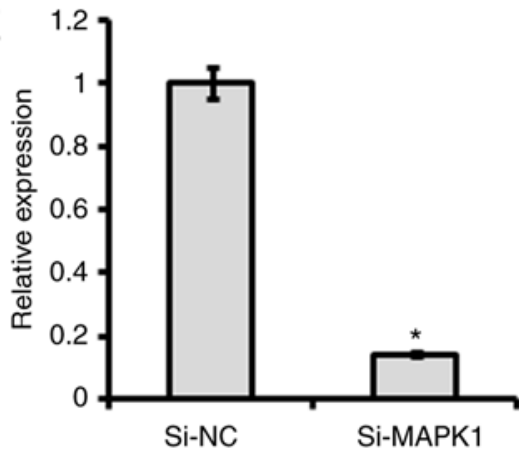

G
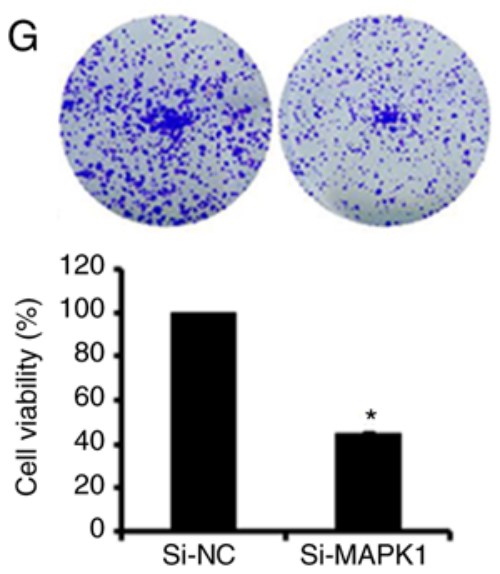

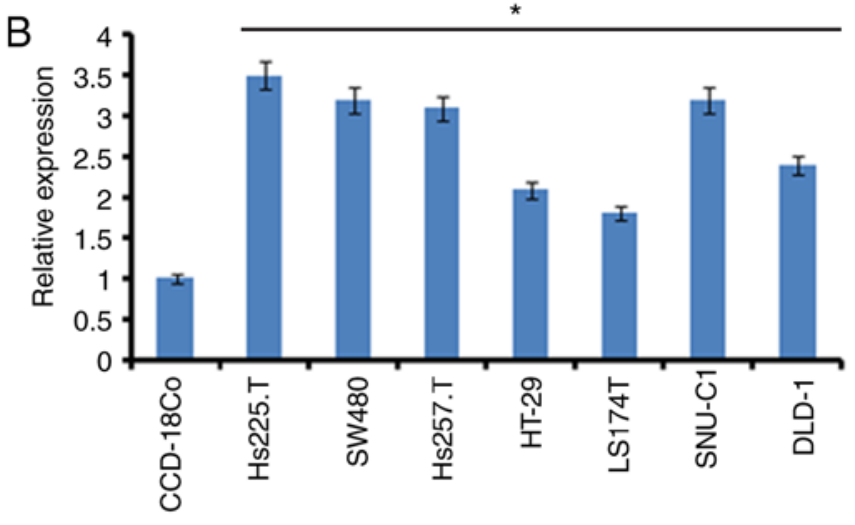

- miR-NC

D $1.2, \square$ miR-145 mimics

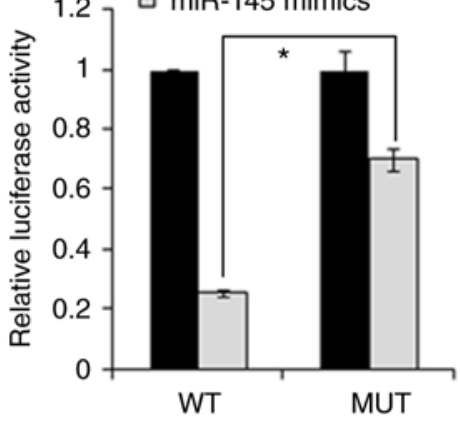

$\mathrm{F}$

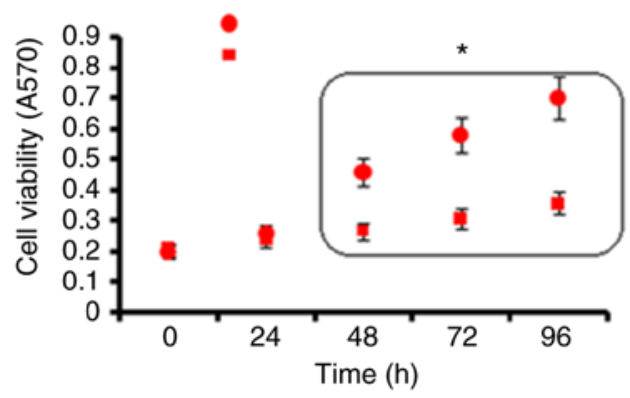

$\mathrm{H}$

Si-NC

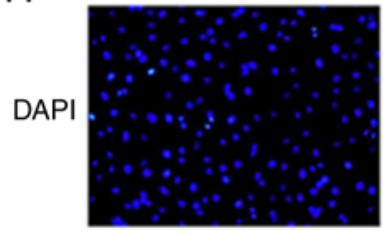

Si-MAPK1
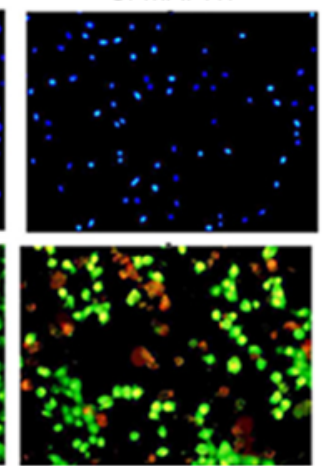

Figure 3. Effects of miR-145 on SW480 cells via MAPK1. (A) Identification of MAPK1 as a potential target of miR-145. (B) Expression of MAPK1 in normal and colon adenocarcinoma cell lines. (C) Expression of MAPK1 in miR-NC- and miR-145 mimics-transfected SW480 cells. (D) Luciferase assay. (E) Expression of MAPK1 in si-NC- or si-MAPK1-transfected SW480 cells. Effect of the suppression of MAPK1 on SW480 cell (F) proliferation, (G) colony formation and $(\mathrm{H})$ apoptosis. The values are expressed as the mean \pm standard deviation of three biological replicates $\left({ }^{*} \mathrm{P}<0.05\right)$. miR, microRNA; NC, negative control; MAPK1, mitogen-activated protein kinase 1; si-, small interfering RNA; WT, wild-type; MUT, mutant (magnification, x200).

in almost all biological pathways (17). Therefore, miRNAs have effects on a wide array of cancer-related processes, which include, but are not limited to, proliferation, metastasis and cell cycle (18). There is substantial evidence relating to the implications of miRNAs in the development of cancer (7). It has been reported that miRNAs are often downregulated in tumors due to a number of factors (8). Consistently, in the present study, it was observed that the expression of miR-145 was significantly downregulated in primary colon adenocarcinoma cells. Furthermore, the ectopic expression of miR-145 inhibited the proliferation of colon adenocarcinoma cells by the induction of apoptosis. The overexpression of miR-145 

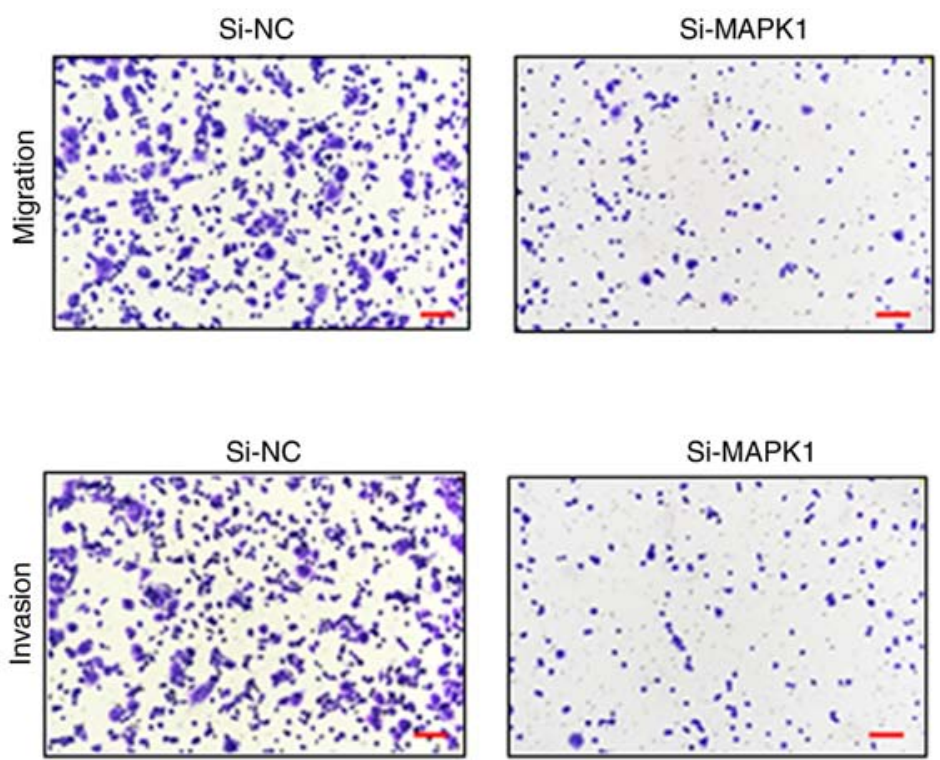
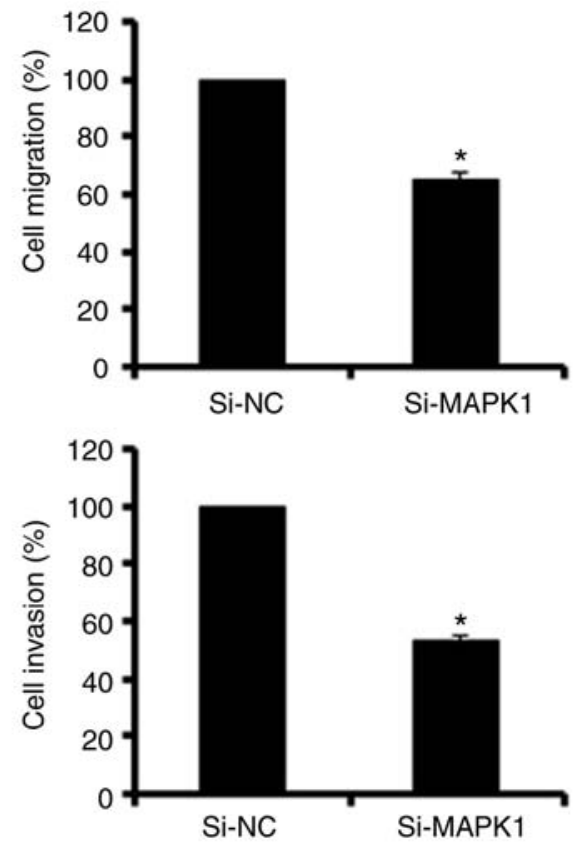

Figure 4. Effect of MAPK1 on the suppression of migration and invasion of SW480 cells. The values are expressed as the mean \pm standard deviation of three biological replicates $\left({ }^{*} \mathrm{P}<0.05\right)$. Scale bars, $100 \mu \mathrm{m} \mathrm{NC}$, negative control; MAPK1, mitogen-activated protein kinase 1; si-, small interfering RNA.

A

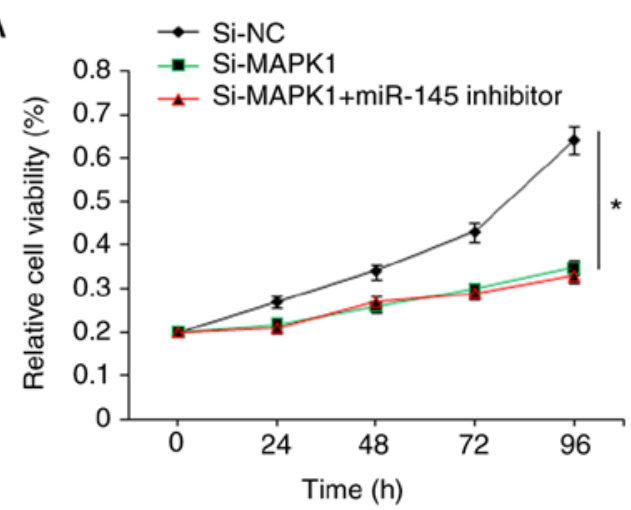

Bi-MAPK1+
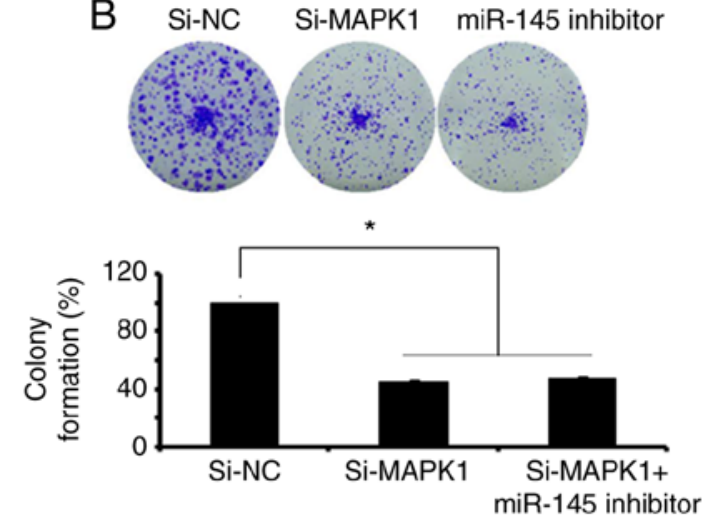

C

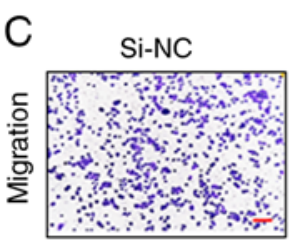

Si-MAPK1

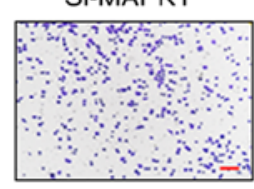

Si-MAPK1+ miR-145 inhibitor
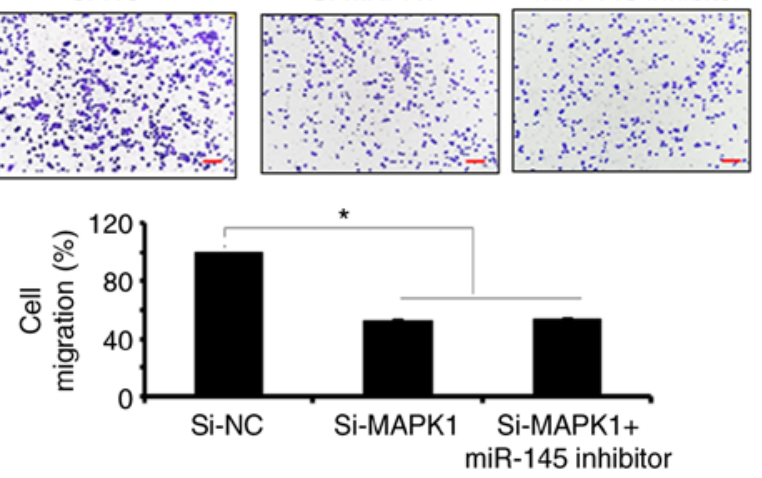
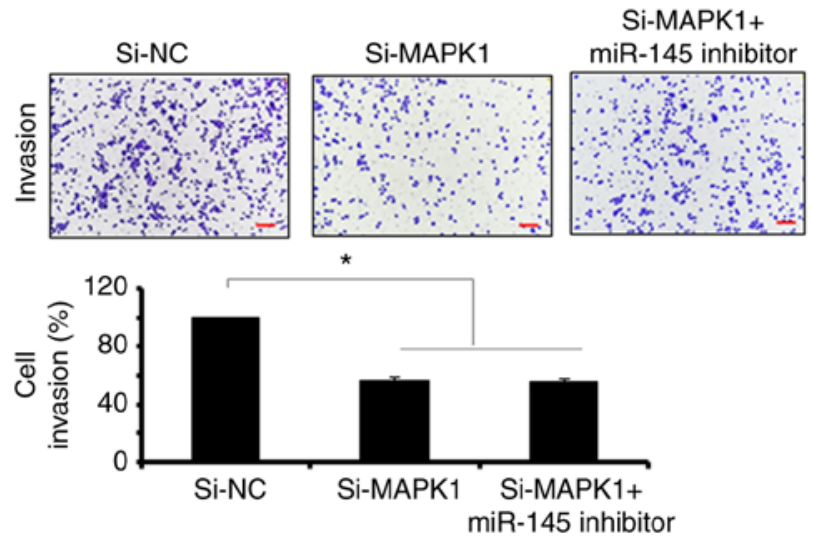

Figure 5. Effect of miR-145 inhibitor on SW480 cells. (A) Proliferation, (B) colony formation, (C) migration and invasion of si-NC- and si-MAPK1-transfected SW480 cells. Scale bars, $100 \mu \mathrm{m}$. The values are expressed as the mean \pm standard deviation of three biological replicates $\left({ }^{*} \mathrm{P}<0.05\right)$. miR, microRNA; NC, negative control; MAPK1, mitogen-activated protein kinase 1; si-, small interfering RNA.

also inhibited the migration and invasion of SW480 colon adenocarcinoma cells. These results are supported by the literature. For example, miR-145 was reported to suppress the proliferation and metastasis of primary colon adenocarcinoma (19). Similarly, Yin et al reported that miR-145 inhibited the growth of lung adenocarcinoma by targeting 

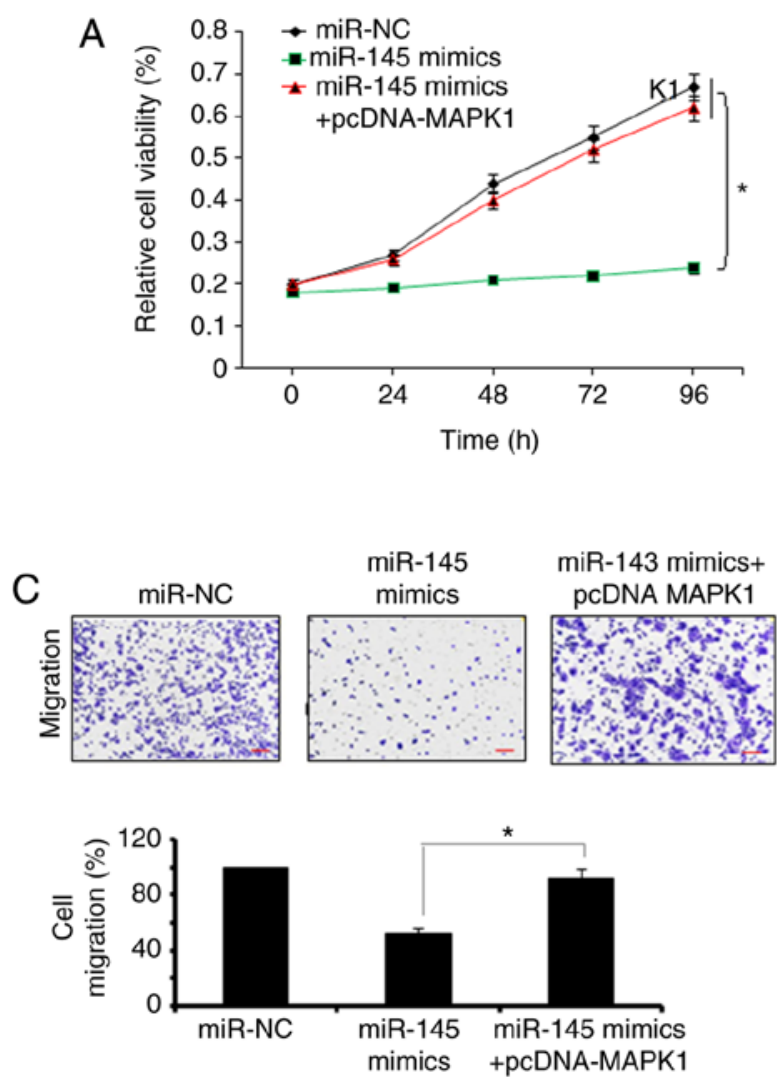
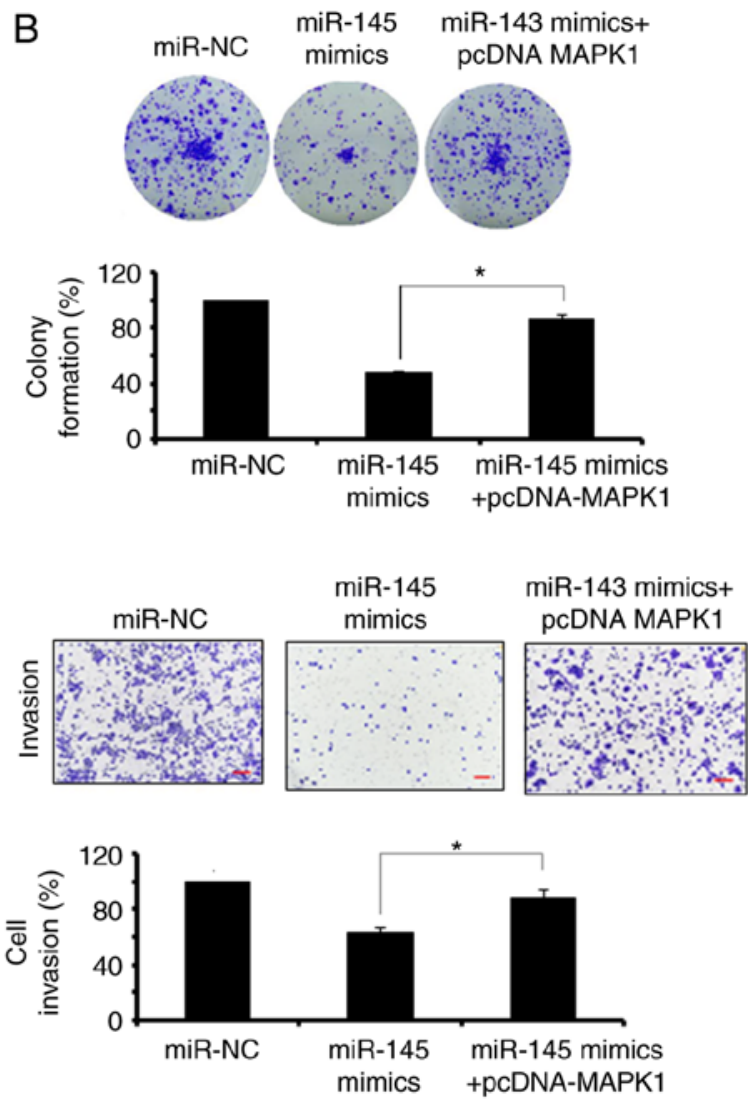

Figure 6. Effect of the overexpression of MAPK1 on SW480 cells. Effect of pcDNA-MAPK1 on (A) proliferation (B) colony formation, (C) migration and invasion of miR-NC- and miR-145 mimics-transfected SW480 cells. The values are expressed as the mean \pm standard deviation of three biological replicates ("P<0.05). miR, microRNA; NC, negative control; MAPK1, mitogen-activated protein kinase 1; pcDNA-MAPK1, overexpression of MAPK1.

A
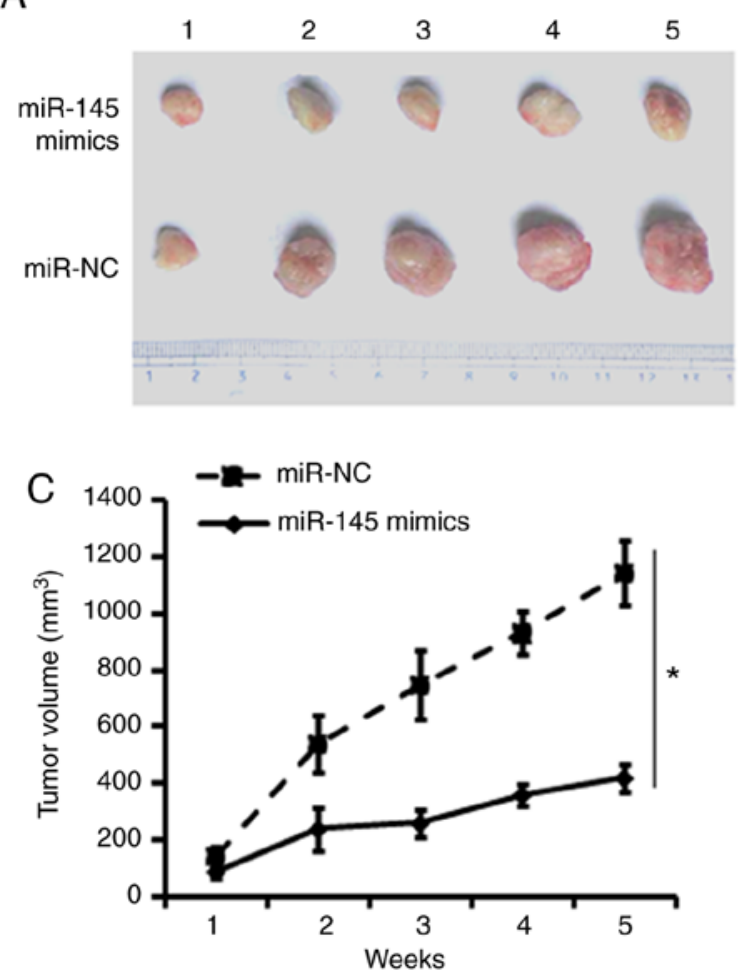
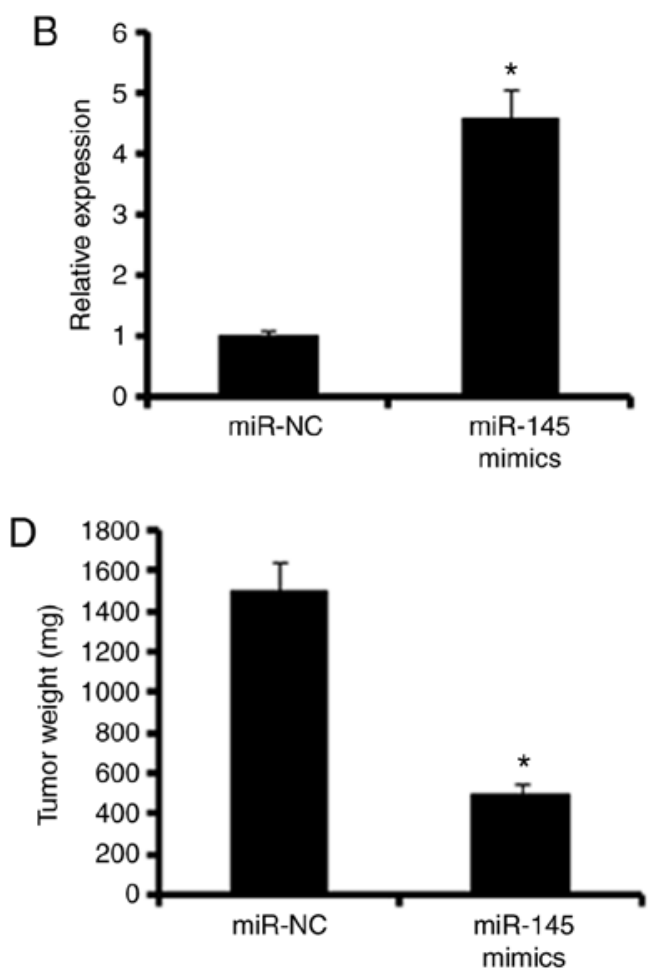

Figure 7. In vivo effects of the overexpression of miR-145 on tumor growth. (A) Images of the miR-NC and miR-145 tumors. (B) Expression of miR-145 in miR-NC and miR-145 mimics xenografted tumors. (C) Effect of miR-NC and miR-145 transfection on xenografted tumor volume. (D) Effect of miR-NC and miR-145 transfection on xenografted tumor weight. Scale bars, $100 \mu \mathrm{m}$. The values are expressed as the mean \pm standard deviation of three biological replicates $\left({ }^{*} \mathrm{P}<0.05\right)$. miR, microRNA; NC, negative control; MAPK1, mitogen-activated protein kinase 1. 
octamer-binding transcription factor 4 (20). Furthermore, miRNA expression studies have revealed the role of miR-145 in the metastasis of colorectal cancer (21). In the present study, the overexpression of miR-145 had no apparent effects on the migration and invasion of non-cancerous cells, indicating that the effects of miR-145 are cancer-specific. Investigation of the underlying mechanism revealed that miR-145 exerted its effects by targeting MAPK1, and silencing MAPK1 had similar effects on the proliferation, migration and invasion of primary colon adenocarcinoma cells as the overexpression of miR-145. Although the inhibition of miR-145 did not rescue the effects of MAPK1silencing on the proliferation, migration and invasion of SW480 cells, the overexpression of MAPK1 reversed the effects of miR-145 mimics on the proliferation, migration and invasion of SW480 cells. MAPK1 has also been previously reported to regulate the growth and apoptosis of cancer cells, confirming the results of the present study (22). In addition, Li et al reported that silencing MAPK1 inhibited the proliferation of HeLa cells (23). Furthermore, MAPK1 has been reported to regulate the apoptotic cell death of cancer cells (24), and MAPK1 has been shown inhibit the expression of matrix metalloproteinase (MMP)-2 and MMP-9 in cervical cancer cells, ultimately inhibiting their migration and invasion (25). Finally, the in vivo evaluation in the present study revealed the growth inhibitory effects of the overexpression of miR-145 on the xenografted tumors. Taken together, it was concluded that miR-145 inhibited the proliferation, migration and invasion of primary colon adenocarcinoma cells by targeting MAPK1; therefore, miR-145 may offer therapeutic potential for the management of colon adenocarcinoma.

\section{Acknowledgements}

Not applicable.

\section{Funding}

The study was supported by Shanghai Hospital of Traditional Chinese Medicine (Shanghai, China) (grant no. TCM-1156/2016).

\section{Availability of data and materials}

The datasets used and/or analyzed in the present study are available from the corresponding author on reasonable request.

\section{Authors' contributions}

YY, XJL and PL performed all the experiments. YY and XJL collected the materials and provided instrumental suggestion for the present study. The present study was designed and supervised by XTG. All authors read and approved the final manuscript.

\section{Ethics approval and consent to participate}

The study was approved and supervised by the Ethics Committee of Shanghai Hospital of Traditional Chinese Medicine under approval number ECSHT-AS345/2017.

\section{Patient consent for publication}

Not applicable.

\section{Competing interests}

The authors declare that they have no competing interests.

\section{References}

1. Schetter AJ, Leung SY, Sohn JJ, Zanetti KA, Bowman ED, Yanaihara N, Yuen ST, Chan TL, Kwong DL, Au GK, et al: MicroRNA expression profiles associated with prognosis and therapeutic outcome in colon adenocarcinoma. Jama 299: 425-436, 2008.

2. Hanif R, Qiao L, Shiff SJ and Rigas B: Curcumin, a natural plant phenolic food additive, inhibits cell proliferation and induces cell cycle changes in colon adenocarcinoma cell lines by a prostaglandin-independent pathway. J Lab Clin Med 130: 576-584, 1997.

3. Hong J, Lu H, Meng X, Ryu JH, Hara Y and Yang CS: Stability, cellular uptake, biotransformation, and efflux of tea polyphenol (-)-epigallocatechin-3-gallate in HT-29 human colon adenocarcinoma cells. Cancer Res 62: 7241-7246, 2002.

4. Tsukuda K, Tanino M, Soga H, Shimizu N and Shimizu K: A novel activating mutation of the K-ras gene in human primary colon adenocarcinoma. Biochem Biophys Res Commun 278: 653-658, 2000.

5. Ambros V: The functions of animal microRNAs. Nature 431: 350-355, 2004.

6. Bartel DP: MicroRNAs: Target recognition and regulatory functions. Cell 136: 215-233, 2009.

7. Esquela-Kerscher A and Slack FJ: Oncomirs-microRNAs with a role in cancer. Nat Rev Cancer 6: 259-269, 2006.

8. Ji W, Sun B and Su C: Targeting microRNAs in cancer gene therapy. Genes (Basel) 8: pii: E21, 2017.

9. Sachdeva M and Mo YY: miR-145-mediated suppression of cell growth, invasion and metastasis. Am J Transl Res 2: 170-180, 2010.

10. Fei B and Wu H: MiR-378 inhibits progression of human gastric cancer MGC-803 cells by targeting MAPK1 in vitro. Oncol Res 20: 557-564, 2013

11. Steibel JP, Poletto R, Coussens PM and Rosa GJ: A powerful and flexible linear mixed model framework for the analysis of relative quantification RT-PCR data. Genomics 94: 146-152, 2009.

12. Vidya Priyadarsini R, Senthil Murugan R, Maitreyi S, Ramalingam K, Karunagaran D and Nagini S: The flavonoid quercetin induces cell cycle arrest and mitochondria-mediated apoptosis in human cervical cancer (HeLa) cells through p53 induction and NF- $\mathrm{B}$ inhibition. Eur J Pharmacol 649: 84-91, 2010.

13. Kasibhatla S, Amarante-Mendes GP, Finucane D, Brunner T, Bossy-Wetzel E and Green DR: Acridine orange/ethidium bromide (AO/EB) staining to detect apoptosis. CSH Protoc 2006: pii:pdb-rot4493, 2006.

14. Wang Y, Zhao W and Fu Q: miR-335 suppresses migration and invasion by targeting ROCK1 in osteosarcoma cells. Mol Cell Biochem 384: 105-111, 2013.

15. Gotham SM, Fryer PJ and Paterson WR: The measurement of insoluble proteins using a modified Bradford assay. Anal Biochem 173: 353-358, 1988.

16. Fhaner CJ, Liu S, Ji H, Simpson RJ and Reid GE: Comprehensive lipidome profiling of isogenic primary and metastatic colon adenocarcinoma cell lines. Anal Chem 84: 8917-8926, 2012.

17. Bushati N and Cohen SM: microRNA functions. Annu Rev Cell Dev Biol 23: 175-205, 2007.

18. Alvarez-Garcia I and Miska EA: MicroRNA functions in animal development and human disease. Development 132: 4653-4662, 2005.

19. Gao S, Wang P, Hua Y, Xi H, Meng Z, Liu T, Chen Z and Liu L: ROR functions as a ceRNA to regulate Nanog expression by sponging miR-145 and predicts poor prognosis in pancreatic cancer. Oncotarget 7: 1608-1618, 2016.

20. Yin R, Zhang S, Wu Y, Fan X, Jiang F, Zhang Z, Feng D, Guo X and Xu L: microRNA-145 suppresses lung adenocarcinomainitiating cell proliferation by targeting OCT4. Oncol Rep 25: 1747-1754, 2011. 
21. Arndt GM, Dossey L, Cullen LM, Lai A, Druker R, Eisbacher M, Zhang C, Tran N, Fan H, Retzlaff K, et al: Characterization of global microRNA expression reveals oncogenic potential of miR-145 in metastatic colorectal cancer. BMC Cancer 9: 374, 2009.

22. Fang JY and Richardson BC: The MAPK signalling pathways and colorectal cancer. Lancet Oncol 6: 322-327, 2005

23. Li XW, Tuergan M and Abulizi G: Expression of MAPK1 in cervical cancer and effect of MAPK1 gene silencing on epithelial-mesenchymal transition, invasion and metastasis. Asian Pac J Trop Med 8: 937-943, 2015.

24. Lwin WW, Park K, Wauson M, Gao Q, Finn PW, Perkins D and Khanna A: Systems biology approach to transplant tolerance: Proof of concept experiments using RNA interference (RNAi) to knock down hub genes in Jurkat and HeLa cells in vitro. J Surg Res 176: e41-e46, 2012.
25. Li XW, Tuergan M and Abulizi G: Expression of MAPK1 in cervical cancer and effect of MAPK1 gene silencing on epithelial mesenchymal transition, invasion and metastasis. Asian Pac J Trop Med 8: 937-943, 2015.

This work is licensed under a Creative Commons Attribution-NonCommercial-NoDerivatives 4.0 International (CC BY-NC-ND 4.0) License. 